\title{
Water-soluble multiwall-carbon-nanotube-polythiophene composite for bilayer photovoltaics
}

\author{
Anthony J. Miller, ${ }^{\text {a) }}$ Ross A. Hatton, and S. Ravi P. Silva \\ Nano-electronics Centre, Advanced Technology Institute, University of Surrey, Guildford, \\ Surrey GU2 7XH, United Kingdom
}

(Received 5 June 2006; accepted 31 July 2006; published online 20 September 2006)

\begin{abstract}
A water-soluble acid oxidized multiwall carbon nanotube (o-MWCNTs)-polythiophene composite for bilayer photovoltaics is reported. Discrete heterojunction photovoltaic cells utilizing this nanocomposite material as the donor layer exhibit a $\sim 20 \%$ increase in fill factor and commensurate increase in power conversion efficiency as compared to cells without o-MWCNTs. Crucially o-MWCNTs are incorporated into the cell structure using an environmentally compatible solvent without complicating the process of device fabrication. (C) 2006 American Institute of Physics. [DOI: $10.1063 / 1.2356115]$
\end{abstract}

In recent years a great deal of research effort has focused on the development of organic and hybrid organic/inorganic photovoltaics. ${ }^{1}$ The principal motivation is to produce a low cost, renewable energy source which will help to solve the world's looming energy crisis. Progress towards achieving the power conversion efficiencies required for commercial viability has been rapid. ${ }^{1}$ However, today's most efficient devices-which are invariably processed from solutionrequire the use of environmentally damaging solvents in the fabrication process. ${ }^{2,3}$

In this letter we report a nanocomposite comprising a homogeneous blend of acid oxidized multiwall carbon nanotubes (o-MWCNTs) and the water-soluble polythiophene, sodium poly[2-(3-thienyl)-ethoxy-4butylsulfonate] (PTEBS). The utility of this material as the donor layer in discrete heterojunction photovoltaic cells employing a $\mathrm{C}_{60}$ acceptor is also reported. In this context, o-MWCNTs facilitate the extraction of positive charge carriers, reducing cell series resistance and increasing cell fill factor, resulting in a significant increase in power conversion efficiency as compared to cells without o-MWCNTs. Importantly, water is used as the solvent to process the nanocomposite which greatly increases the environmental compatibility of the fabrication process. Furthermore, o-MWCNTs are integrated into the cell structure without complicating the process of cell fabrication.

Water-soluble thiophene was purchased from American Dye Source Inc. ${ }^{4}$ Multiwall carbon nanotubes ( $>90 \%$ nanotube purity) grown by chemical vapor deposition were obtained commercially (Nanocyl) and used as received. Stable dispersions of o-MWCNTs in deionized water were prepared by ultrasonically dispersing carbon nanotubes in a 3:1 mixture of concentrated sulfuric and nitric acids and refluxing at $130{ }^{\circ} \mathrm{C}$ for $60 \mathrm{~min}$ before diluting with HPLC grade deionized water. The resulting dispersion was centrifuged and the acid supernatant decanted off. The remaining solid was repeatedly washed with HPLC grade water over a $50 \mathrm{~nm}$ polycarbonate filter until the washings were $p \mathrm{H} \mathrm{6-7.} \mathrm{To} \mathrm{deter-}$ mine the o-MWCNTs loading, a known volume of the stock solution was completely dried and weighed. The typical diameter and length of the o-MWCNTs after acid treatment

${ }^{a)}$ Electronic mail: anthony.miller@surrey.ac.uk were confirmed using transmission electron microscopy (TEM) to be $10 \mathrm{~nm}$ and several microns, respectively.

All devices were fabricated on indium tin oxide (ITO) coated glass (Merck) with a nominal sheet resistance of $20 \Omega \mathrm{sq}^{-1}$. ITO substrates were cleaned by sonicating in toluene, an aqueous solution of Decon-90, acetone, and finally refluxing acetone vapor. Substrates were microwave oxygen plasma treated before film deposition. o-MWCNTs were mixed in $1 \%, 3.5 \%$, and $7.5 \%$ by weight (wt. $\%$ ) with PTEBS in $0.1 M$ ammonia solution to aid solubility. The PTEBS concentration used was $7.5 \mathrm{mg} \mathrm{ml}^{-1}$ and was mixed with the appropriate wt. \% of o-MWCNTs. The solution was spin coated onto the ITO substrates at $700 \mathrm{rpm}$ and then heated in air at $100{ }^{\circ} \mathrm{C}$ for $10 \mathrm{~min}$ to expel residual water and ammonia. $\mathrm{C}_{60}(99.9 \%)$ was deposited in a vacuum system to a thickness of $50 \mathrm{~nm}$. Without breaking the vacuum a $50 \mathrm{~nm}$ aluminum layer was evaporated through a shadow mask to form the top electrode. Film thickness was monitored using a quartz crystal microbalance and the photoactive device area was typically $7.5-10 \mathrm{~mm}^{2}$. Devices were tested using an Oriel solar simulator under AM1.5D spectral illumination of $100 \mathrm{~mW} \mathrm{~cm}^{-2}$ (1 sun). The output power of the simulator was measured using a calibrated thermopile. All measurements were made in air immediately after device fabrication. Atomic force microscope (AFM) measurements were made under ambient conditions.

Achieving stable concentrated dispersions of MWCNTs in solution requires that they are chemically functionalized. ${ }^{5}$ o-MWCNTs readily form concentrated aqueous dispersions with PTEBS which can be spin cast onto ITO glass to form thin films of uniform thickness (as determined by atomic force microscopy). Since o-MWCNTs are electrically conducting, it is essential that they are confined within the donor layer to avoid short circuiting the device. AFM studies of spin coated films of the nanocomposite confirm that the o-MWCNTs do not significantly protrude from the film surface [Fig. 1(a)]. Furthermore, the roughness of PTEBS and PTEBS/o-MWCNTs composite films is comparable for all o-MWCNTs loadings investigated, evidence that o-MWCNTs are uniformly distributed and of the compatibility between PTEBS and o-MWCNTs. Scanning electron microscopy (SEM) images acquired using an electron beam energy sufficient to probe the entire film thickness [Fig. 1(b)] 

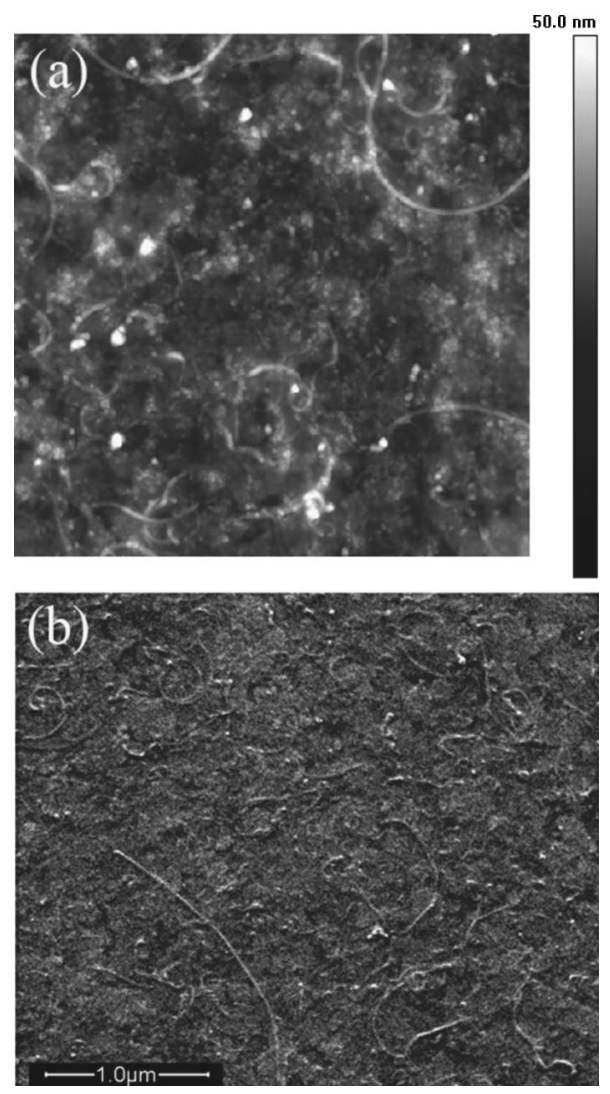

FIG. 1. Representative AFM $\left(3 \times 3 \mu \mathrm{m}^{3}\right)$ (a) and SEM (b) images of PTEBS/o-MWCNTs (7.5 wt. \%) composite films.

confirm that the o-MWCNTs are uniformly distributed with the PTEBS layer.

The absorption spectra of PTEBS, o-MWCNTs, and PTEBS/o-MWCNTs composite between 200-1200 nm are shown in Fig. 2. The absorption spectra of PTEBS can be modified by changing the $p \mathrm{H}$ of the solution. ${ }^{6}$ When the polymer is dissolved in water, absorption is confined to the region below $600 \mathrm{~nm}$ having a maximum at $435 \mathrm{~nm}$ which corresponds to the $\pi$ - $\pi^{*}$ electronic transition. Upon reducing the $p \mathrm{H}$ an additional peak appears at $773 \mathrm{~nm}$ and the solution changes from orange to dark green. The o-MWCNTs absorb throughout the measured energy range with a peak maximum at $\sim 280 \mathrm{~nm}$ assigned to the $\pi-\pi^{*}$ optical transition from the density of states previously identified by Ago et al. ${ }^{7}$ The composite spectra are the superposition of the o-MWCNTs and PTEBS spectra, indicating the absence of significant ground state electronic or charge transfer interactions be-

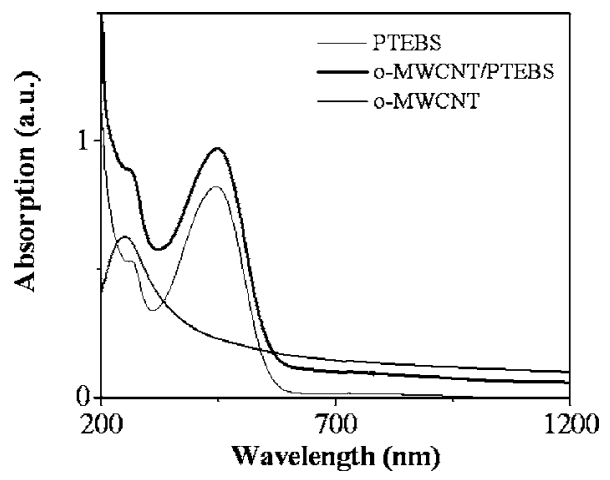

FIG. 2. Absorption spectra between 200 and $1200 \mathrm{~nm}$ of PTEBS, o-MWCNTs, and PTEBS/o-MWCNTs in water. Downloaded 31 Mar 2009 to 131.227.178.132. Redistribution subject to AlP license or copyright; see http://apl.aip.org/apl/copyright.jsp
TABLE I. Summary of device characteristics under 1 sun simulated solar illumination.

\begin{tabular}{ccccc}
\hline \hline o-MWCNTs (wt. \%) & $V_{\mathrm{oc}} \pm 0.03$ & $J_{\mathrm{sc}}\left(\mathrm{mA} \mathrm{cm}^{-2}\right)$ & FF & PCE \\
\hline 0 & 0.65 & 1.40 & 0.51 & 0.46 \\
1 & 0.62 & 1.49 & 0.61 & 0.56 \\
3.5 & 0.60 & 1.49 & 0.59 & 0.52 \\
7.5 & 0.57 & 1.52 & 0.62 & 0.55 \\
\hline \hline
\end{tabular}

tween the two materials under low white light intensities. The absence of a new peak at $773 \mathrm{~nm}$ also indicates that the PTEBS is not, to any significant extent, doped by protons associated with the acid functionality on o-MWCNTs.

Table I summarizes values for open circuit voltage $\left(V_{\mathrm{oc}}\right)$, short circuit current density $\left(J_{\mathrm{sc}}\right)$, fill factor $(\mathrm{FF})$, and power conversion efficiency (PCE) for cells with and without o-MWCNTs dispersed within the PTEBS donor layer. Inclusion of o-MWCNTs within the device structure improves cell fill factor by $\sim 20 \%$ resulting in a commensurate increase in PCE. Notably the increase in cell FF remains constant over the full range of o-MWCNTs loadings investigated herein. Both $J_{\mathrm{sc}}$ and $V_{\mathrm{oc}}$ are almost unchanged upon incorporation of o-MWCNTs.

The large increase in FF upon incorporation of o-MWCNTs results from a reduction in cell series resistance, which is evident as an increase in the gradient of the photoresponse where it intersects the $x$ axis (Fig. 3). The cell series resistance is the sum of contributions from bulk transport within the organic layers, carrier extraction at the electrodes, and transport through the electrodes to the external circuit. Since the highest occupied molecular orbital (HOMO) of PTEBS (5.0-5.3 eV) (Ref. 8) and the Fermi level of o-MWCNTs (5.0-5.1 eV, Ref. 7 and this work) are well aligned, it is likely that the highly conductive o-MWCNTs facilitate hole transport through the PTEBS layer to the ITO electrode.

AFM measurements of PTEBS and PTEBS/o-MWCNTs films [Fig. 1(a)] show that the roughness is not significantly changed upon incorporation of o-MWCNTs into the PTEBS layer for all of the loadings investigated. Consequently, the small increase in $J_{\mathrm{sc}}$ (Table I) cannot be attributed to an

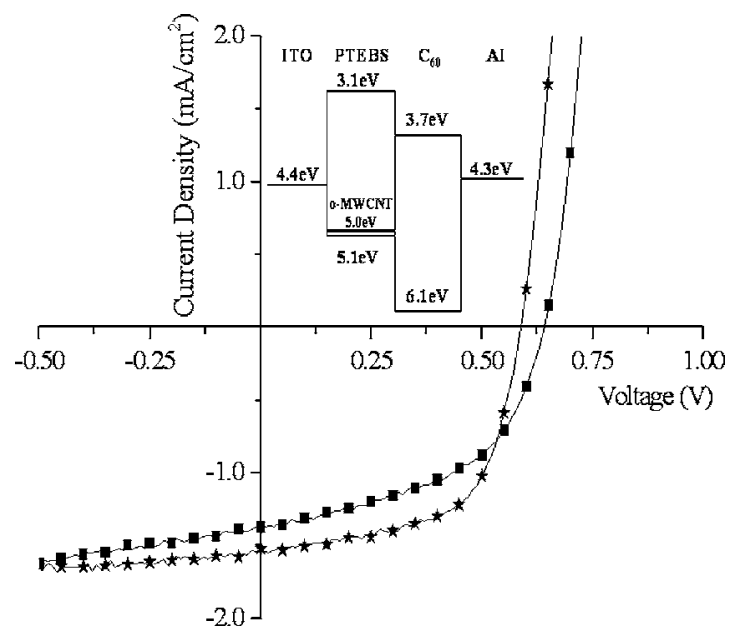

FIG. 3. Typical current-voltage characteristics of cells with 7.5 wt. $\%$ o-MWCNTs (stars) and without o-MWCNTs (squares) dispersed within the PTEBS layer. Inset: schematic energy level diagram of device structure 
increase in the PTEBS/ $\mathrm{C}_{60}$ interfacial area. MWCNTs are known to quench photoluminescence in conjugated polymers, which would be expected to reduce $J_{\text {sc }}$. However, $J_{\text {sc }}$ in cells incorporating o-MWCNTs is actually slightly increased. One possible explanation is that o-MWCNTs do not reduce the photocurrent by quenching excitons formed in the PTEBS prior to dissociation, since the HOMO of PTEBS and the Fermi level of o-MWCNTs are well aligned. Alternatively it may indicate that each o-MWCNTs is coated with a PTEBS thickness greater than twice the singlet state exciton diffusion length, which is typically less than $10 \mathrm{~nm}$ in semiconducting polymers. ${ }^{9}$

There are two possible explanations for the very small reduction in $V_{\text {oc }}$. It has recently been shown that $V_{\text {oc }}$ in bilayer organic solar cells is virtually independent of the work function of the hole-extracting electrode. ${ }^{10}$ In this case the work functions of the microwave oxygen plasma treated ITO electrode and o-MWCNTs were measured using ultraviolet photoelectron spectroscopy (UPS) to be $\sim 4.4$ and $\sim 5.0 \mathrm{eV}$, respectively. Such a small change in $V_{\text {oc }}$ would be consistent with this idea. An alternative and more likely explanation is that the o-MWCNTs are completely coated with PTEBS preventing direct contact with the underlying ITO electrode. However, since the Fermi level of o-MWCNTs and the HOMO of PTEBS are well aligned, o-MWCNTs facilitate conduction, rather than trapping, holes on their path to the ITO electrode.

Figure 3 shows the typical current-voltage characteristics under 1 sun simulated solar illumination of cells with and without the maximum o-MWCNTs loading of $7.5 \mathrm{wt} \%$ within the PTEBS layer, from which it is clear that the cell shunt resistance is virtually unchanged upon incorporation of o-MWCNTs. This is further evidence that o-MWCNTs do not contribute to leakage currents across the device. Figure 3 (inset) illustrates the proposed energy level diagram for a PTEBS:o-MWCNT/C 60 photovoltaic device based on UPS measurements of the microwave oxygen plasma treated ITO glass and o-MWCNTs work functions-4.4 and $5.0 \mathrm{eV}$, respectively - and the previously reported HOMO and low- est unoccupied molecular orbital energies of PTEBS (Ref. 8) and $\mathrm{C}_{60}{ }^{11}$

In addition to the excellent potential o-MWCNTs have to increase the power conversion efficiency of organic solar cells, we anticipate that they may also enhance the mechanical strength of these devices, particularly for relatively high o-MWCNTs loadings such as those used herein. This is an increasingly relevant property with the growing tendency towards fully conformable devices on flexible substrates.

In summary we have fabricated discrete heterojunction solar cells using an o-MWCNTs:PTEBS nanocomposite donor layer and $\mathrm{C}_{60}$ acceptor layer. Inclusion of o-MWCNTs within the PTEBS donor increases cell FF by $\sim 20 \%$ resulting in a commensurate increase in PCE. This improvement in performance is attributed to a reduction in cell series resistance resulting from improved hole transport within the PTEBS layer. Crucially, this composite material is processed from an aqueous solution making it environmentally compatible with low cost fabrication for large area devices.

The authors would like to acknowledge Nicholas P. Blanchard for the UPS measurements and Vlad Stolojan for the TEM measurements. We would also like to thank the EPSRC for funding via a Portfolio Partnership award.

${ }^{1}$ S. Shaheen, D. Ginley, and G. Jabbour, MRS Bull. 30, 10 (2005).

${ }^{2}$ L. Gang, V. Shrotriya, J. Huang, Y. Yao, T. Moriarty, K. Emery, and Y. Yang, Nat. Mater. 4, 864 (2005).

${ }^{3}$ M. Wanli, Y. Cuiying, G. Xiong, L. Kwanghee, and A. Heeger, Adv. Funct. Mater. 15, 1617 (2005).

${ }^{4}$ American Dye Source, http://www.adsdyes.com/products/pdf/ polythiophene/ADS2000P.pdf

${ }^{5}$ S. Sinnott, J. Nanosci. Nanotechnol. 2, 113 (2002).

${ }^{6}$ F. Tran-Van, M. Carrier, and C. Chevrot, Synth. Met. 142, 251 (2004).

${ }^{7}$ H. Ago, T. Kugler, F. Cacialli, W. Salaneck, M. Shaffer, A. Windle, and R. Friend, J. Phys. Chem. B 103, 8116 (1999).

${ }^{8}$ Q. Qiao, L. Su, J. Beck, and J. McLeskey, J. Appl. Phys. 98, 1094906 (2005).

${ }^{9}$ P. Peumans, A. Yakimov, and S. Forrest, J. Appl. Phys. 93, 1 (2003).

${ }^{10}$ S. Khodabakhsh, B. Sanderson, J. Nelson, and T. Jones, Adv. Funct. Mater. 16, 95 (2006).

${ }^{11}$ Organic Photovoltaics Concepts and Realization, edited by C. Brabec, V. Dyakonov, J. Parisi, and N. S. Sariciftci (Springer, Berlin, 2003), p. 165 . 